HEALTHCARE DELIVERY

\title{
Women's willingness to use emergency contraception: Experience at Chris Hani Baragwanath Academic Hospital, Johannesburg, South Africa
}

\author{
T A Lukhaimane, Y Adam \\ Tshimangadzo Lukhaimane, MB ChB, FCOG (SA), MMed, has a particular interest in diabetes and other endocrinopathies in pregnancy, and \\ Yasmin Adam, BSc, MB BCh, FCOG (SA), MSc (Biostatistics and Epidemiology), an interest in the prevention of cervical cancer and in contraception. \\ They are specialists in the Department of Obstetrics and Gynaecology, Chris Hani Baragwanath Academic Hospital, Johannesburg, South Africa.
}

Corresponding author: Y Adam (yasminadam@gmail.com)

Access to emergency contraception (EC) has little restriction in South Africa. EC is a contraceptive method that can be used by women up to 7 days after unprotected intercourse. It can be used in the following situations: when no contraceptive has been used; for condom accidents; after intrauterine contraceptive device expulsion; when a contraceptive method has been incorrectly used, or contraceptive pills missed; if there has been a $>3$-hour delay in taking the progestogen-only pill, a $>2$-week delay for intramuscular depot medroxyprogesterone acetate or a >1-week delay for intramuscular norethisterone enanthate; or after delayed placement or early removal or dislodgement of a contraceptive transdermal patch or vaginal ring.

S Afr Med J 2015;105(4):266-267. DOI:10.7196/SAMJ.9411

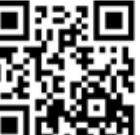

Emergency contraception (EC) is available from healthcare providers in South Africa (SA) without a prescription. However, awareness of EC is as low as $35.5 \%$ in SA (while being as high as $94 \%$ in a UK population). ${ }^{[1,2]}$ Women younger than 20 years of age, with a higher level of education and who spoke either English or Afrikaans were more aware of EC. ${ }^{[1,3]}$ Uptake of EC has been seen to increase when dedicated products are available, as seen in France and Nigeria. ${ }^{[4]}$ Knowledge of EC among healthcare providers may also impact on use of the method. In Durban only a third of pharmacists and doctors could correctly initiate an EC regimen. ${ }^{[5]}$ There were misconceptions that EC was an abortifacient, or an agent that might promote promiscuity, HIV and sexually transmitted infections. ${ }^{[5,6]}$

One hundred women who had delivered at Chris Hani Baragwanath Academic Hospital, Johannesburg, SA, were interviewed to explore their awareness and knowledge of and willingness to use EC. Ethics approval was obtained from the Human Research Ethics Committee at the University of the Witwatersrand, Johannesburg (ethics clearance certificate No. M120354).

\section{Awareness and knowledge of EC}

Forty-four women were aware of EC. Most $(28,63.6 \%)$ had heard of EC from friends or peers, $9(20.5 \%)$ at a local clinic, 2 (4.5\%) from educators, 2 (4.5\%) from family, 1 (2.3\%) from a pharmacy, and 2 (4.5\%) from multiple sources. Awareness of EC was significantly associated with being single, having a senior secondary or tertiary education, being employed and being born in SA. Of those who were aware, 34 (77.3\%) knew the correct time that EC could be used, 42 (95.5\%) knew where it could be obtained, and 30 (68.2\%) knew that it was available over the counter from a healthcare provider. Two women $(4.5 \%)$ believed that a prescription was required, and the remaining 12 (27.3\%) were not sure. Repeated use of EC was reported as being possible by 11 women (25.0\%), and $23(52.3 \%)$ were not sure. Four women $(9.1 \%)$ correctly identified side-effects of EC, 24 (54.5\%) believed that there were no side-effects, and 15 (34.1\%) were unsure whether there were side-effects ( 1 woman did not give an answer). Only 1 woman $(2.3 \%)$ was aware that an intrauterine contraceptive device could be used as EC. The other 43 (97.7\%) knew of EC as a pill formulation.

\section{Willingness to use EC}

The 44 women were given a leaflet on EC that explained when and how it can be taken, how to access it, the time at which it should be taken, the types of EC available, where it can be accessed, the mechanism of action and the side-effects. Their willingness to use EC was then assessed. Thirty-five (79.5\%) of the women reported that they had not recognised an opportunity to use EC in the past, while $9(20.4 \%)$ had recognised an opportunity to utilise EC and had done so.

The initial sample of 100 women were asked about their willingness to use EC. The majority ( 85 women) expressed a willingness to use EC, while 14 were not willing and 1 was unsure. The concerns that women raised were personal and related to stigma and religion. Four women had no preference regarding where they would obtain EC, 63 cited the local clinic, 30 would use a private pharmacy, 1 would go to a general practitioner and 2 would approach either a pharmacy or a general practitioner.

The 100 women were then asked whether or not the concept of an advance supply of EC was something that they considered favourable, and whether they had any concerns regarding an advance supply: 70 supported an advance supply of EC, while 29 were against it and 1 was undecided.

Twenty-four of the women expressed concerns with the concept of an advance supply, citing potential for abuse, promotion of promiscuity (especially by the youth), incorrect use, absence of medical advice, wastage of unused medication, and deliberate misuse of regular contraception. A larger proportion (43 women) had positive comments regarding an advance supply of EC, such as convenience of access, no need to queue or worry that a health facility would be closed, privacy and anonymity, no need to face negative staff attitudes, promotion of responsible behaviour, 
control over reproductive choice, and affordability. Thirty-three women did not give an answer.

Most respondents (63 women) said they would prefer to obtain EC from their local clinic for reasons of convenience and affordability. Those who preferred a doctor or pharmacy highlighted issues such as stigmatisation by clinic staff, long queues, healthcare clinics being closed over weekends or limited access as reasons for opting for sources that they acknowledged would incur a cost.

\section{Discussion}

The low awareness of EC among the women surveyed is a concern, particularly as most of them had booked antenatally (98.0\%) and had therefore had some interaction with the health services. Of those who were aware, at least two-thirds knew the correct source of access, the correct timing of use and the fact that EC is available without a prescription. Other studies in SA have shown a 7\% awareness of EC in HIV-infected women on antiretroviral therapy, ${ }^{[7]}$ $22.8 \%$ awareness among women attending clinics in Gauteng ${ }^{[8]}$ and $35-60 \%$ awareness among women in a termination of pregnancy clinic. ${ }^{[1,8]}$

In developed countries where EC awareness is high, a lack of knowledge of the potential risk of pregnancy after condom slippage or missed contraceptive pills ${ }^{[9]}$ accounts for underutilisation. Willingness to use the method was high $(85.0 \%)$ once women were given the knowledge, highlighting the need for better access to information.

Women's concerns about an advance supply of EC are relevant and would have to be considered in SA, especially as a randomised control trial of an advance supply v. none in advance among postpartum women in the USA showed that an advance supply increased use of EC (relative risk 4.0). ${ }^{[10]}$

Significantly, only $20.4 \%$ of the women who were aware of EC had learned about it at a public healthcare facility, and none had heard of it during antenatal visits. Rather, knowledge of EC was gained from non-healthcare sources, which may explain women's poor depth of knowledge.

\section{Conclusion}

We have identified that EC awareness is lacking and knowledge is poor among women accessing the public sector. If it is freely available but underutilised, EC is unable to make a positive impact on women's health and fertility regulation. All contraceptive counselling should include a discussion of EC, incorporating advice on an advance supply.

Disclosure of interests. This article was submitted to the University of the Witwatersrand by TAL as part of her MMed dissertation.

Authorship. TAL conceived the study on which the article is based, wrote the protocol and submitted it to the ethics committee. She also performed all the interviews, entered the data into an Excel spreadsheet and wrote the dissertation for her MMed. YA supervised the project from protocol to dissertation and analysed the data.

Funding. No funding was required.

1. Moodley J, Morroni C. Emergency contraception - lack of awareness among women presenting for termination of pregnancy. S Afr Med J 2007;97(8):584-585. [http://www.ncbi.nlm.nih.gov/pubmed/17952213]

2. Lakha F, Glasier A. Unintended pregnancy and use of emergency contraception among a large cohort of women attending for antenatal care or abortion in Scotland. Lancet 2006;368(9549):1782-1787. [http://dx.doi.org/10.1016 S0140-6736(06)69737-7]

3. Roberts C, Moodley J, Esterhuizen T. Emergency contraception: Knowledge and practices of tertiary students in Durban, South Africa. J Obstet Gynaecol 2004;24(4):441-445. [http://dx.doi.org/10.1080/01443610410001685619]

Marston C Cleland J. Do unintended pregnancies carried to term lead to adverse outcomes for mother and child? An assessment in five developing countries. Popul Stud (Camb) 2003;57(1):77-93. [http://dx.doi.org/ child? An assessment in five devel
$10.1080 / 0032472032000061749]$

5. Blanchard K, Harrison T, Sello M. Pharmacists' knowledge and perceptions of emergency contraceptive pills in Soweto and the Johannesburg Central Business District, South Africa. Int Fam Plan Perspect
. pills in Soweto and the Johannesburg Central Business District, South Afrit
2005;31(4):172-178. [http://www.guttmacher.org/pubs/journals/3117205.html]

6. Golden NH, Seigel WM, Fisher M, et al. Emergency contraception: Pediatricians' knowledge, attitudes, and opinions. Pediatrics 2001;107(2):287-292. [http://pediatrics.aappublications.org/content/107/2/287.long]

7. Myer L, Rebe K, Morroni C. Missed opportunities to address reproductive health care needs among HIVinfected women in antiretroviral therapy programmes. Trop Med Int Health 2007;12(12):1484-1489. [http:// dx.doi.org/10.1111/j.1365-3156.2007.01955.x]

8. Siebert I, Steyn PS. Knowledge and use of emergency contraception in a tertiary referral unit in a developing country. Eur J Contracept Reprod Health Care 2002;7(3):137-143. [http://dx.doi.org/10.1080/ejc.73.137.143] Ibisomi LDG, Odimegwu C. Predictors of unintended pregnancy among South African youth. East Afr Soc Sci Res Rev 2007;23(1):61-80.

10. Jackson R, Schwarz EB, Freedman L, Darney P. Knowledge and willingness to use emergency contraception among low-income post-partum women. Contraception 2000;61(6):351-357. [http://dx.doi.org/10.1016/ S0010-7824(00)00117-7]

Accepted 2 February 2015. 